\title{
Effect of Preoperative Uric Acid Level and Neutrophil / Lymphocyte Ratio on Preoperative and Postoperative Visual Analogue Pain Scores in Patients with Lumbar Disc Herniation: A Cross-Sectional Study
}

\author{
Huseyin BOZKURT ${ }^{1}$, Densel ARAC ${ }^{2}$, Burhanettin CIGDEM $^{3}$ \\ ${ }^{1}$ Cumhuriyet University, Faculty of Medicine, Department of Neurosurgery, Sivas, Turkey \\ ${ }^{2}$ Necmettin Erbakan University, Meram Faculty of Medicine, Department of Neurosurgery, Konya, Turkey \\ ${ }^{3}$ Cumhuriyet University, Faculty of Medicine, Department of Neurology, Sivas, Turkey \\ Corresponding author: Huseyin BOZKURT bozkurthuseyinn@gmail.com
}

\section{ABSTRACT}

AIM: To determine the relationship between the serum urate (SU) level, neutrophil / lymphocyte ratio (NLR), and pain severity using preoperative and postoperative visual analogue scale (VAS) scores in patients with lumbar disc herniation (LDH).

MATERIAL and METHODS: This single-center, cross-sectional study included 20 consecutive patients who were operated for LDH by the same surgeon. The patients'pre- and postoperative UA levels, NLRs, and intensity severity VAS scores were investigated. Preoperative magnetic resonance imaging (MRI) findings, serum UA levels, and neutrophil and lymphocyte counts were recorded. Pain severity was recorded preoperatively and at 6 months postoperatively. Effects of the preoperative SU levels and NLRs on the pre- and postoperative VAS scores were statistically assessed.

RESULTS: Statistically significant positive correlation coefficients were determined between NLR and the preoperative and postoperative VAS scores. Negative correlation coefficients were found between the SU levels and preoperative VAS scores; in contrast, positive correlation coefficients were found between the SU levels and the postoperative VAS scores.

CONCLUSION: Our results demonstrate the importance of not ignoring the serum UA level and NLR in pre- and postoperative pain in patients with LDH. Nevertheless, further extensive studies are warranted.

KEYWORDS: Lumbar disc herniation, Serum urate, Neutrophil/lymphocyte ratio

ABBREVIATIONS: SU: Serum urate, NLR: Neutrophil / lymphocyte ratio, VAS: Visual analogue scale, LDH: Lumbar disc herniation, UA: uric acid, MRI: Magnetic resonance imaging

\section{INTRODUCTION}

umbar disc herniation (LDH) is the leading cause of lower back and leg pain. LDH may clinically manifest as dull Jor sharp pain in the lower back or lower extremities, muscle spasms, sciatica, paresthesia, and muscle weakness in the lower extremities (10). Uric acid (UA) is the final waste product of purine metabolism. Under normal conditions, UA occurs in the serum as urate ions; at high concentrations, it becomes insoluble, such as in gout disease. An excess of UA in the serum is known as hyperuricemia (4). UA is an essential antioxidant in human plasma, and its increase in an intracellular environment has pro-oxidant properties. Increased serum urate (SU) levels induce oxidative stress-related disorders, such as obesity, hypertension, cardiovascular diseases, renal failure, fibromyalgia, and chronic musculoskeletal pains that 
cannot be diagnosed (15). Decreased SU levels have been associated with multiple sclerosis (MS), Parkinson's disease, Alzheimer's disease, and optic neuritis. Although beneficial to organisms under normal levels, SU becomes destructive at levels higher than normal (12).

Neutrophil / lymphocyte ratio (NLR) is one of the several inflammatory parameters used as prognostic markers to determine the systemic inflammatory response. NLR is an inexpensive and easily obtainable inflammatory marker and is calculated using the neutrophil and lymphocyte levels obtained from the complete blood count (1). Increased NLR has been investigated in several diseases, such as fibromyalgia, autoimmune diseases, various malignancies, infections, metabolic syndrome, cardiovascular diseases, hypertension, chronic obstructive pulmonary disease, preeclampsia, eclampsia, major depression, and mood disorders.

Several studies in the literature have investigated the effects of SU levels and NLRs on cardiovascular, renal, and neurological diseases as well as on cancer. Through this study, we aimed to reveal the significance of SU levels and NLRs in LDH, which is the most commonly treated disease in the field of neurosurgery.

\section{- MATERIAL and METHODS}

\section{Study Population}

This cross-sectional study was conducted on 20 consecutive patients who underwent surgery for LDH at a single center. The study was approved by the Human Research Ethics Committee of our university. Written informed consent was obtained from all participants prior to study initiation.

Patients with magnetic resonance imaging (MRI) and magnetic resonance myelography findings, pain and painful symptoms ongoing for 12 months, preoperative radiological diagnosis of L4-L5 disc herniation with pain or numbness in lumbar dermatomal distribution, motor or sensory neurological signs (hypesthesia, hyperesthesia, allodynia, or dysesthesia) in the affected dermatomes, sufficient cognitive ability to participate in the study, and neurogenic intermittent claudication were included in the study. Patients with rheumatoid arthritis, known peripheral neuropathies, spondylolisthesis, renal insufficiency, monologue narrative of LSS surgeon, cancer, acute and/or chronic systemic diseases (cardiac diseases, peripheral vascular disease, and/or diabetes mellitus), chronic depression or antidepressant use, acute and/ or chronic inflammatory systemic and/or local diseases, and LDH at any other level except L4-L5 were excluded.

The age, sex, neutrophil and lymphocyte counts, serum UA levels, and lumbar MRI data were recorded for all participants. Blood samples were collected from the antecubital vein in the morning hours. Venous blood samples were collected into tubes with and without an anticoagulant. NLR was calculated using the neutrophil and lymphocyte levels obtained from the complete blood count. The patients' visual analogue scale (VAS) scores were recorded preoperatively and at 6 months postoperatively.

\section{Statistical Analysis}

Data were analyzed using IBM SPSS for Windows version 22.0 software (SPSS Inc.; Chicago, IL, USA). The Kolmogorov-Smirnov and Wilcoxon signed-rank tests were used for evaluating data that did not meet the parametric test assumptions. Correlation coefficients were calculated using Spearman analysis, and 0.05 was considered as the level of error. $\mathrm{p}<0.05$ was considered statistically significant.

\section{RESULTS}

The study comprised 14 (70\%) females and 6 (30\%) males; the median patient age was 47.5 years (mean, $46.45 \pm 13.50$ years; range, $25-74$ years). The body mass index values, socio economic levels, disc hernia levels, and disc hernia volumes on lumbar MRI were similar in all the patients without any other chronic or acute systemic or inflammatory diseases (Table I). A statistically significant difference was found between the preoperative and postoperative VAS score measurements $(p<0.05$; Table II). Surgical treatment was indeed beneficial, with evident psychological differences in the mobilization stage.

Statistically significant positive correlation coefficients $(r)$ were determined between NLR and the preoperative VAS scores ( $r: 0.472$ ) and between NLR and the postoperative VAS scores $(r: 0.570)(p<0.05)$. When the NLR increased, the VAS score also increased (Table III). The increased VAS scores were associated with the inflammatory effect of NLR accurately explained postoperative patient satisfaction and preoperative intolerable pain.

Negative correlation coefficients were found between the SU levels and preoperative VAS scores ( $r$ : -0.125$)$, and positive correlation coefficients were found between the SU levels and postoperative VAS scores ( $r$ : 0.091). These values were small and not statistically significant (Table IV).

\section{DISCUSSION}

Manipulation of the serum UA levels is promising in the treatment of several diseases. Based on the fact that both decreased and increased UA levels reportedly contribute to the development and progression of several diseases, any significant changes in the UA levels should be imminently addressed and normalized. Abnormally high UA levelsare associated with gout, hypertension, cardiovascular diseases, and renal diseases, where as low UA levels are associated with MS, Parkinson's disease, Alzheimer's disease, and optic neuritis. However, it is unclear whether low serum UA levels are the cause or result of these neuro degenerative diseases. Individuals with low serum UA levels may not be able to prevent free radical toxicity, inflammation development, and tissue destruction. Furthermore, it has been observed that excessive use of UA by the free radicals produced during inflammation occurring in MS may lead to even lower UA levels (6).

One of the most important areas where in the antioxidant effects of UA are beneficial is in the central nervous system, particularly in MS, Parkinson's disease, and acute stroke 
Table I: Laboratory Parameters and Characteristics of the Patients

\begin{tabular}{|c|c|c|c|c|c|c|c|}
\hline \multirow[t]{2}{*}{ Patient \# } & \multirow[t]{2}{*}{ Age } & \multirow[t]{2}{*}{ Gender } & \multirow[t]{2}{*}{ Serum Urate $(\mathrm{mg} / \mathrm{dL})$} & \multirow[t]{2}{*}{ Neu / Lym } & \multirow[t]{2}{*}{ NLR } & \multicolumn{2}{|c|}{ VAS } \\
\hline & & & & & & Preoperative & Postoperative \\
\hline 1 & 47 & $\mathrm{~F}$ & 3.9 & $2.41 / 2.74$ & 0.87 & 9 & 1 \\
\hline 2 & 50 & $\mathrm{~F}$ & 5 & $4.55 / 2.47$ & 1.84 & 9 & 2 \\
\hline 3 & 42 & $\mathrm{~F}$ & 5.2 & $4.44 / 2.2$ & 2.01 & 7 & 1 \\
\hline 4 & 58 & $\mathrm{~F}$ & 3.7 & 4.69 / 2.36 & 1.98 & 8 & 0 \\
\hline 5 & 52 & $\mathrm{~F}$ & 4.2 & $3.15 / 2.17$ & 1.45 & 7 & 7 \\
\hline 6 & 48 & $\mathrm{~F}$ & 4.6 & $8.18 / 2.58$ & 3.17 & 10 & 6 \\
\hline 7 & 51 & M & 3.3 & $3.26 / 1.37$ & 2.37 & 9 & 1 \\
\hline 8 & 37 & M & 3.9 & $7.61 / 2.17$ & 3.5 & 10 & 5 \\
\hline 9 & 33 & $\mathrm{~F}$ & 2 & $8.8 / 1.14$ & 7.7 & 10 & 3 \\
\hline 10 & 74 & $\mathrm{~F}$ & 6.1 & $3.27 / 2.55$ & 1.28 & 8 & 0 \\
\hline 11 & 63 & M & 6.2 & $3.88 / 1.22$ & 3.18 & 9 & 4 \\
\hline 12 & 25 & M & 5.8 & $4.64 / 1.7$ & 2.72 & 10 & 3 \\
\hline 13 & 64 & $\mathrm{~F}$ & 2.4 & $3.82 / 2.17$ & 1.76 & 8 & 2 \\
\hline 14 & 29 & $\mathrm{~F}$ & 3.2 & $5.05 / 1.53$ & 3.3 & 8 & 2 \\
\hline 15 & 30 & $M$ & 4.1 & 3.7 / 2.71 & 1.36 & 7 & 1 \\
\hline 16 & 48 & M & 8 & 4 / 2.04 & 1.36 & 8 & 2 \\
\hline 17 & 32 & $\mathrm{~F}$ & 4 & $4.47 / 1.95$ & 2.29 & 8 & 8 \\
\hline 18 & 44 & $\mathrm{~F}$ & 3.9 & $2.75 / 2.64$ & 1.04 & 9 & 0 \\
\hline 19 & 38 & $\mathrm{~F}$ & 3 & 3.79 / 2.61 & 1.45 & 9 & 1 \\
\hline 20 & 64 & $\mathrm{~F}$ & 5.1 & 4.32 / 1.99 & 2.17 & 9 & 1 \\
\hline
\end{tabular}

NEU / LYM: Neutrophil / lymphocyte, NLR: Neutrophil / lymphocyte ratio, VAS: Visual analogue scale.

Table II: Statistical Analysis of Pre-and Postoperative VAS Score of Study Patients

\begin{tabular}{lccc}
\hline VAS & Mean & Std. Deviation & $\mathbf{p}$ \\
\hline Preoperative & 8.60 & 0.99 & $\mathbf{0 . 0 0 1}$ \\
\hline Postoperative & 2.50 & 2.35 & \\
\hline
\end{tabular}

VAS: Visual analogue scale.

Table III: Statistical Analysis of Neutrophil / Lymphocyte Ratio on Pre- and Post-Operative VAS Scores

NLR VAS

\begin{tabular}{ccc}
\hline & Preoperative & Postoperative \\
\hline $\mathrm{r}$ & 0.472 & 0.570 \\
$\mathrm{p}$ & $\mathbf{0 . 0 3 6}$ & $\mathbf{0 . 0 0 9}$ \\
\hline $\mathrm{n}$ & 20 & 20 \\
\hline
\end{tabular}

VAS: Visual analogue scale, NLR: Neutrophil / lymphocyte ratio.
Table IV: Statistical Analysis of Serum Urate Levels on Pre- and Post-Operative VAS Scores

\begin{tabular}{cccc}
\hline Serum Urate & \multicolumn{2}{c}{ VAS } \\
\hline & Preoperative & Postoperative \\
\hline $\mathrm{r}$ & -0.125 & 0.091 \\
\hline $\mathrm{p}$ & 0.600 & 0.704 \\
\hline $\mathrm{n}$ & 20 & 20 \\
\hline
\end{tabular}

VAS: Visual analogue scale.

$(2,7,11,17)$. Although chronic increased UA levels have been associated with an increased stroke risk, acute increases in UA levels can protect against some antioxidants. A previous experimental study reported that UA protects against oxidative stress in cultured rat hippocampal neuronal cells and that the application of UA 24 hours before middle cerebral artery obstruction reduced brain damage induced by acute ischemia (25). The preventive properties of UA against the 
harmful effects of peroxynitrite on spinal cord neurons were confirmed by adding UA at different concentrations to primary spinal cord neuron cultures and evaluating the effects on mitochondrial respiration and $\mathrm{LDH}$ oscillation. UA reportedly provides significant protection to the spinal cord neurons against the toxic effects of peroxynitrite in a dose-dependent manner; protection was provided even at UA doses as low as $100 \mu \mathrm{M}(16)$. In addition to the effects on neurons, UA reportedly decreases neutrophil infiltration and hepatic injury during hemorrhagic shock (22). However, despite these benefits of UA, higher rates of cardiovascular and all-cause mortality have been reported in patients with high levels of UA than in those with normal levels (20). Although the pathogenesis of these diseases is extremely complex and unclear, all of them have been observed to have the paradoxical commonality of oxidative stress and oxidative modifications of proteins and lipids $(5,24)$.

These findings suggest that UA can act as an antioxidant owing to its ability to prevent the acute activation of proinflammatory cells in the blood by oxidants (15). A Norwegian study involving 1500 individuals aged 40-42 years reported that widespread "weather-dependent" pain was associated with increased SU levels, and a Swedish population-based study involving1609 individuals aged 25-74 years confirmed that the extent of pain was related to the level of SU (3). Therefore, the serum UA level can be considered as a marker of disease status. Moreover, recent studies have provided evidence that UA may play a substantial role in the development or progression of these disease types. Although some changes in UA levels could be due to the disease, the levels may play a significant role in the development and prevention of several diseases. Therefore, it has been clarified that UA, which was previously considered an inert organic component, could play significant roles in several biological functions (12). UA can be beneficial both as an antioxidant and a free radical scavenger; however, it can become harmful at high levels. In conclusion, the manipulation of UA levels is currently included in the treatment of various diseases or is being investigated.

In the present study, when SU level was low, the preoperative and postoperative VAS scores were found to be high and low, respectively. According to previous studies, the abovementioned finding may be attributable to SU levels, with their antioxidant effect, being the parameter that resulted in increased pain in postoperative patients and reduced pain in preoperative patients; however, this finding was not statistically significant.

NLR is a prognostic marker used to determine systemic inflammatory responses $(8,19,23)$; as such, NLR has been studied in systemic disorders with increased neutrophil and decreased lymphocyte counts during inflammation. Increased blood NLR is also reportedly associated with increased disease activity in many systemic, rheumatological, and neurological diseases as well as in cancers. Lymphocyte reduction, physiological stress, and acute inflammation are reflected by NLR $(14,18,21)$.
NLR is the ratio between cells mediating two different immune pathways. Neutrophils represent the first line of immune defense, exhibiting phagocytic and apoptotic actions via the secretion of various inflammatory mediators, particularly cytokines (13). Inflammation triggered by cytokines can induce further inflammation owing to cell dysfunction and oxidative stress. In contrast, lymphocytes are specific inflammatory mediators; they have a regulatory or protective function, and a low lymphocyte count reflects poor general health and physiological stress (9).

In concordance with the literature, our results indicate that the preoperative and postoperative pain were higher in patients with a high NLR, which is an indicator of inflammation. Moreover, it can be considered that relevant results could be obtained with multicentre studies involving more patients.

\section{CONCLUSION}

$\mathrm{LDH}$ is a widespread disease with an increasing incidence; it also has several associated etiological factors. Recent studies have shown that inflammation plays a role in the increase of pain severity. This study evaluated the preoperative UA level, NLR, and pre- and post-operative VAS scores of patients undergoing surgery for LDH. The main finding of this study was the significant relationship between NLR and the preoperative and postoperative VAS scores of the patients who were operated. This situation can be considered for a failed back surgery where in the preoperative NLRs of patients with LDH are controlled to provide an increased level of satisfaction after surgery. Another advantage of this study is that the markers evaluated are inexpensive and can be rapidly assessed using the results obtained from complete blood count tests. To the best of our knowledge, this is the first study in the literature that has examined the effect of SU levels and NLRs on satisfaction of and pain severity in patients undergoing surgery for LDH. However, future studies are required to further confirm and clarify the present results.

\section{REFERENCES}

1. Akturk S, Buyukavci R: Evaluation of blood neutrophillymphocyte ratio and platelet distribution width as inflammatory markers in patients with fibromyalgia. Clin Rheumatol 36(8):1885-1889, 2017

2. Amaro S, Soy D, Obach V, Cervera A, Planas AM, Chamorro A: A pilot study of dual treatment with recombinant tissue plasminogen activator and uric acid in acute ischemic stroke. Stroke 38: 2173-2175, 2007

3. Andersson HI, Ejlertsson G, Leden I, Rosenberg C: Characteristics of subjects with chronic pain, in relation to local and widespread pain report-a prospective study of symptoms, clinical findings and blood tests in subgroups of a geographically defined population. Scand J Rheumatol 25:146-154,1996

4. Andersson HI, Leden I: Increased serum uric acid-a marker of non-gouty widespread pain? A study of female patients with inflammatory and non-inflammatory pain. Scand J Rheumatol 35(4):261-267, 2006 
5. Berg AH, Lin Y, Lisanti MP, Scherer PE: Adipocyte differentiation induces dynamic changes in NF-kappaB expression and activity. Am J Physiol Endocrinol Metab 287:1178-1188, 2004.

6. Drulovic J, Dujmovic I, Stojsavljevic N, Mesaros S, Andjelkovic S, Miljkovic D, Peric V, Dragutinovic G, Marinkovic J, Levic Z, Mostarica Stojkovic M: Uric acid levels in sera from patients with multiple sclerosis. J Neurol 248:121-126, 2001

7. Duan W, Ladenheim B, Cutler RG, Kruman II, Cadet $\mathrm{JL}$, Mattson MP: Dietary folate deficiency and elevated homocysteine levels endanger dopaminergic neurons in models of Parkinson's disease. J Neurochem 80:101-110, 2002.

8. Galizia G, Lieto E, Zamboli A, De Vita F, Castellano P, Romano C, Auricchio A, Cardella F, De Stefano L, Orditura M: Neutrophil to lymphocyte ratio is a strong predictor of tumor recurrence in early colon cancers: A propensity score-matched analysis. Surgery 158(1):112-120, 2015

9. Gibson $\mathrm{PH}$, Cuthbertson BH, Croal BL, Rae D, El-Shafei H, Gibson G, Jeffrey RR, Buchan KG, Hillis GS: Usefulness of neutrophil/lymphocyte ratio as predictor of new-onset atrial fibrillation after coronary artery bypass grafting. Am $\mathrm{J}$ Cardiol 105(2):186-191, 2010

10. Hao DJ, Duan K, Liu TJ, Liu JJ, Wang WT: Development and clinical application of grading and classification criteria of lumbar disc herniation. Medicine (Baltimore) 96(47):e8676, 2017

11. Hooper DC, Spitsin S, Kean RB, Champion JM, Dickson GM, Chaudhry I, Koprowski H: Uric acid, a natural scavenger of peroxynitrite, in experimental allergic encephalomyelitis and multiple sclerosis. Proc Natl Acad Sci USA 95:675-680, 1998

12. Kutzing MK, Firestein BL: Altered uric acid levels and disease states. J Pharmacol Exp Ther 324:1-7, 2008

13. Mayadas TN, Cullere $X$, Lowell CA: The multifaceted functions of neutrophils. Annu Rev Pathol 9:181-218, 2014

14. Proctor MJ, McMillan DC, Morrison DS, Fletcher CD, Horgan PG, Clarke SJ: A derived neutrophil to lymphocyte ratio predicts survival in patients with cancer. $\mathrm{Br} \mathrm{J}$ Cancer 107(4):695-699, 2012

15. Sautin YY, Johnson RJ: Uric acid: The oxidant-antioxidant paradox. Nucleosides Nucleotides Nucleic Acid 27:608-619, 2008
16. Scott GS, Cuzzocrea S, Genovese T, Koprowski H, Hooper DC: Uric acid protects against secondary damage after spinal cord injury. Proceedings of the National Academy of Sciences 102: 3483-3488, 2005

17. Spitsin SV, Scott GS, Mikheeva T, Zborek A, Kean RB, Brimer CM, Koprowski H, Hooper DC: Comparison of uric acid and ascorbic acid in protection against EAE. Free Radic Biol Med 33:1363-1371, 2002

18. Tamhane UU, Aneja S, Montgomery D, Rogers EK, Eagle KA, Gurm HS: Association between admission neutrophil to lymphocyte ratio and outcomes in patients with acute coronary syndrome. Am J Cardiol 102(6): 653-657, 2008

19. Tasoglu I, Cicek OF, Lafci G, Kadirogullari E, Sert DE, Demir A, Cavus U, Colak N, Songur M, Hodo B: Usefulness of neutrophil/lymphocyte ratio as a predictor of amputation after embolectomy for acute limb ischemia. Ann Vasc Surg 28(3):606-613, 2014

20. Tomita M, Mizuno S, Yamanaka H, Hosoda Y, Sakuma K, Matuoka Y, Odaka M, Yamaguchi M, Yosida H, Morisawa $\mathrm{H}$, Murayama T: Does hyperuricemia affect mortality? A prospective cohort study of Japanese male workers. J Epidemiol 10:403-409, 2000

21. Torun S, Tunc BD, Suvak B, Yildiz H, Tas A, Sayilir A, Ozderin $\mathrm{YO}$, Beyazit $\mathrm{Y}$, Kayacetin E: Assessment of neutrophillymphocyte ratio in ulcerative colitis: A promising marker in predicting disease severity. Clin Res Hepatol Gastroenterol 36:491-497, 2012

22. Tsukada K, Hasegawa T, Tsutsumi S, Katoh H, Kuwano H, Miyazaki T, Yamamoto Y: Effect of uric acid on liver injury during hemorrhagic shock. Surgery 127:439-446, 2000

23. Uslu AU, Deveci K, Korkmaz S, Aydin B, Senel S, Sancakdar E, Sencan M. Is neutrophil / lymphocyte ratio associated with subclinical inflammation and amyloidosis in patients with familial Mediterranean fever? Biomed Res Int 2013:185317, 2013

24. Wellen KE, Hotamisligil GS: Inflammation, stress, and diabetes. J Clin Invest 115: 1111- 1119, 2005

25. Yu ZF, Bruce-Keller AJ, Goodman Y, Mattson MP: Uric acid protects neurons against excitotoxic and metabolic insults in cell culture, and against focal ischemic brain injury in vivo. $J$ Neurosci Res 53:613-625, 1998 